\title{
Nicht jede ICS/LABA-Kombination wirkt gleich gut
}

— Wer wiederholt schwere AsthmaExazerbationen erleidet, riskiert langfristig einen fortschreitenden Verlust der Lungenfunktion [Bai TR et al. Eur Resp J. 2007;30:452], berichtete Prof. Christian Virchow, Rostock. Daher gehört es zu den vorrangigen Therapiezielen, Exazerbationen zu verhindern.

Fixkombinationen von inhalativen Steroiden (ICS) mit langwirksamen Betamimetika (LABA) werden in den aktuellen Asthma-Leitlinien ab Stufe 3 als Basistherapie empfohlen. Die verfügbaren ICS/LABA-Fixkombinationen könnten aber Unterschiede bezüglich des Exazerbationsrisikos aufweisen, ergänzte Prof. Alberto Papi, Ferrara. Er präsentierte die Ergebnisse eines eigenen Literaturvergleichs [Papi A et al. J Aerosol Med Pulm Drug Del. 2016;29:346], welche die
Exazerbationsraten unterschiedlicher ICS/LABA-Fixkombinationen verglich. Berücksichtigt wurden zwei offene Beobachtungsstudien mit der Fluticason/Formoterol-Fixkombination flutiform ${ }^{\circledast}$ sowie drei Cochrane-Meta-Analysen zu anderen ICS/LABA-Kombinationen.

Exazerbationen, die orale Steroide (OCS) notwendig machten, traten unter flutiform ${ }^{\circledast}$ mit einer Inzidenz von 2,1\% auf. So niedrige Inzidenzen wurden nur in 2 von 19 Behandlungsarmen beobachtet, die ausgewertet worden waren. Die Kombinationen Fluticason/Salmeterol und Budesonid/Formoterol führten dagegen zu OCS-pflichtigen Exazerbationsraten von ca. 10-15\%.

Zwar handelt es sich um einen indirekten Vergleich unterschiedlicher Studien mit unterschiedlichen Patienten- kollektiven, doch vertrat Papi die Ansicht, dass die Unterschiede auch mit günstigen pharmakologischen und mechanischen Charakteristika der Fluticason/Formoterol-Fixkombination erklärt werden könnten.

Formoterol habe gegenüber Salmeterol den Vorteil des schnelleren Wirkeintritts. Fluticasonpropionat habe in $\mathrm{Re}$ view-Arbeiten eine bessere Wirkung auf $\mathrm{FEV}_{1}$ und symptomfreie Tage im Vergleich zu anderen ICS gezeigt. Hinzu kommt laut Papi bei flutiform ${ }^{\oplus}$ eine gute Deposition der Wirkstoffe in zentralen und peripheren Bereichen des Bronchialbaums.

Dr. Dirk Einecke

- Symposium "Navigating the bronchial tube: connecting the routes for successful real-life outcomes in asthma care", International Congress 2016 der European Respiratory Society; London, September 2016 (Veranstalter: Mundipharma)

\section{Diabetes mellitus}

\section{Alarmsignal Onychomykosen}

_ Nagelmykosen sind eine oft unterschätzte Infektion bei Diabetikern. Das Risiko, Ulzerationen am Fuß zu entwickeln, ist bei Diabetikern in diesem Fall um das 1,6-fache erhöht.

Daher ist bei Diabetespatienten ein unmittelbarer Therapiebeginn indiziert. Für die lokale Therapie sollte ein Antimykotikum mit einem breiten Wirkspektrum gewählt werden.

Patienten mit Diabetes mellitus sollte $\mathrm{zu}$ einer Therapieform geraten werden, bei der es nicht notwendig ist, die Nägel vor der Behandlung dünn zu feilen. Die abgefeilten Nagelspäne sind infektiös und das Verletzungsrisiko beim Hantieren mit der Feile ist groß.
Der Wirkstoff Ciclopirox in wasserlöslicher Lackgrundlage ist in der lokalen Behandlung von Nagelmykosen etabliert. Er hat ein breites Wirkspektrum und besitzt in vitro antibakterielle und antiphlogistische Eigenschaften, die besonders bei unklaren Mischinfektionen von Vorteil sind.

Die Anwendung ist denkbar einfach: Der Nagellack wird abends vor dem $\mathrm{Zu}$ bettgehen auf die betroffenen Nägel und die umliegende Haut aufgepinselt. Innerhalb von sechs Stunden penetriert der Lack bis tief in den Nagel und gelangt so auch an schwer erreichbare Stellen, etwa das Nagelbett. Lackreste können am nächsten Morgen mit Wasser ab-

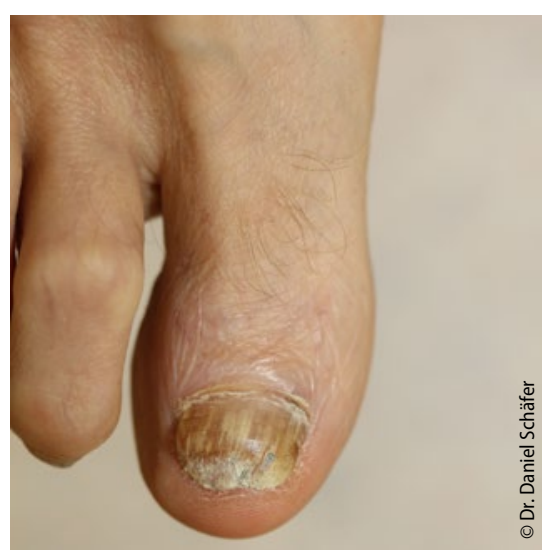

Onychomykosen bei Diabetikern steigern das Risiko, Ulzerationen zu entwickeln.

gewaschen werden. Der Wirkstoff verbleibt im Nagel.

Red.

- Nach Informationen von Taurus 